\title{
Cutting down on prostate cancer metastases
}

Metastatic castration-resistant prostate cancer (mCRPC) carries a poor prognosis, and anti-androgen receptor $(\mathrm{AR})$ therapies - the mainstay of treatment for prostate cancer - are ineffective in these patients. Freeman and colleagues have found that the transcription factor ONECUT2 (OC2) is a potent cell survival factor in these cancers, and that OC2 inhibition in MCRPC mouse models reduces tumour growth and metastasis.

The authors used a previously described resource in which they assembled 38 transcriptome data sets from 2,115 patients with prostate cancer. OC2 was one of the most active transcription factors in this analysis, as determined by expression levels of the transcription factor itself and its target genes.

OC2 is also found in numerous prostate cancer cell lines. Silencing of OC2 using short hairpin RNA (shRNA) induced apoptosis in prostate cancer cell lines. Cells that survived shRNA treatment grew slower in culture and in mouse xenograft models than the parental cell line.

OC2 overexpression in prostate cancer cell lines repressed genes associated with AR activity - most patients with mCRPC have previously received anti-AR therapy and become resistant to it, and these data suggest that OC2 can displace AR-dependent growth and survival mechanisms. OC2 also increased the expression of gene signatures associated with RAS activity and epithelial-mesenchymal transition. In chromatin immunoprecipitation and sequencing studies, OC2 bound to the promoter regions of activated and repressed target genes, implying direct regulation.

OC2 expression correlated with markers of neuroendocrine differentiation; OC2 contains a binding site for REST, a master repressor of neuronal differentiation, which could explain this correlation.

In patients from whom tissue was obtained before therapy, high expression of OC2 mRNA was associated with subsequent biochemical disease recurrence after androgen deprivation therapy, suggesting that OC2 expression could be a biomarker for and contribute to this failure. Furthermore, in these patient cohorts there was an inverse correlation between OC2 activation and AR activation. In another set of patients, OC2-high, AR-low tumours were more prevalent in patients who presented with metastatic disease.

With these data in hand, the authors set out to identify small-molecule inhibitors of OC2 using an in silico chemical screen of 500,000 compounds based on a constructed 3D model of the HOX domain of OC2. The resulting lead compound, CSRM617, inhibited growth and induced apoptosis in many prostate cancer cell lines, and inhibited the transcription of genes identified as part of the OC2 transcriptional signature in their initial analysis.

CSRM617 was then tested in a xenograft model using an mCRPC cell line (22Rv1). Treatment of mice with an intraperitoneal injection of $50 \mathrm{mg}$ per $\mathrm{kg}$ per day of CSRM617, started once the tumours reached $200 \mathrm{~mm}^{3}$, significantly reduced tumour volume. CSRM617 also decreased prostate cancer metastases: the compound reduced the onset and growth of luciferasetagged 22Rv1 cells that were injected intracardially and subsequently found as diffuse metastases. Finally, in mice with established 22Rv1 metastases, treatment with CSRM617 reduced the size and number of detectable luciferasepositive metastases.

Taken together, these results suggest that OC2 is a critical regulator of mCRPC cell growth, particularly in metastatic lesions. The smallmolecule OC2 inhibitor CSRM617 could be used as a lead candidate to develop therapeutics for mCRPC.

\section{Megan Cully}

ORIGINAL ARTICLE Rotinen, M. et al. ONECUT2 is a targetable master regulator of lethal prostate cancer that suppresses the androgen axis. Nat. Med. 24, 1887-1898 (2018) 